\title{
L'hybridation in situ en cytogénétique
}

Grâce à l'hybridation in situ, la cytogénétique, c'est-à-dire l'examen des chromosomes, est devenue moléculaire. Il est maintenant possible de localiser directement un gène sur un chromosome, ce qui a déjà d'importantes implications pour le diagnostic de maladies congénitales ou cancéreuses.

\section{Marie-Geneviève Mattei \\ Chargée de recherche Cnrs \\ Jean-François Mattei \\ Professeur de génétique médicale à la faculté. \\ Médecin des Hôpitaux}

Remerciements

Nous remercions Jean-Louis Mandel (Strasbourg, $\mathrm{U}_{184}$ ) de nous avoir aimablement fourni les sondes $D_{X S} 5_{2}, F$ IX et $D_{1} 8 S_{3}$, ainsi que Kay Davies (Oxford) pour la sonde DXS 5 I. Nous remercions Édith Passage pour sa collaboration technique.

\section{ADRESSE}

M. G. Mattci, J. F. Mattei : Inserm U 242 et Centre de génétique médicale, Hôpital d'enfants de la Timone, $133^{85}$ Marseille Cedex 5.

$\mathrm{m} / \mathrm{s} n^{0}$ I vol. 2 janvier 86
- hybridation in situ (HIS) chromosomique permet de localiser sur des chromosomes métaphasiques la ou les molécule(s) d'ADN complémentaire(s) d'une séquence donnée d'acides nucléiques. Cette technique, réalisée pour la première fois en 1969 sur des chromosomes d'amphibiens [I], fut appliquée à l'homme quelques années plus tard pour localiser les séquences d' $A D N$ satellite* $^{*}[2,3]$. Néanmoins, jusqu'en I98I, l'utilisation de l'HIS était limitée, soit à la localisation de séquences d'ADN répétées un grand nombre de fois dans le génome, soit à la localisation de séquences uniques sur les chromosomes tout à fait particuliers que sont les chromosomes polytènes* de certaines espèces animales. Les progrès techniques réalisés, comme l'utilisation de sondes clonées et l'adjonction de sulfate de dextran à la solution d'hybridation [4] permettent actuellement de localiser par HIS n'importe quelle séquence unique d'une taille au moins égale à 500 paires de bases. L'HIS nécessite tout d'abord le marquage radio-actif

- Voir Glossaire page $4 I$. d'une séquence d'ADN ou sonde, puis son hybridation* avec l'ADN chromosomique, et enfin, après autoradiographie, l'identification de la ou des région(s) chromosomique(s) hybridée(s) [5].

\section{Préparation \\ de la sonde}

La sonde purifiée (généralement fragment d'ADN inséré dans un plasmide), est marquée par nick translation* avec des nucléotides tritiés (figure 1 , voir p. 36). L'utilisation du tritium pose moins de problèmes techniques que celle de l'iode I25, et les sondes tritiées ont par ailleurs l'avantage de pouvoir être conservées plus longtemps. L'ADN radio-actif est alors dissous dans une solution contenant différents facteurs destinés à favoriser l'hybridation (formamide, sulfate de dextran...). La sonde est dénaturée à $70^{\circ} \mathrm{C}$ avant la réaction d'hybridation.

\section{Hybridation avec I'ADN chromosomique}

Les préparations chromosomiques sont réalisées selon les procédés habituels. L'incorporation de 5-bromodéoxyuridine $(5 \mathrm{BrdU})$ pendant 


\section{REFERENCES}

I. Gall JG, Pardue ML. Formation and detection of RNA-DNA hybrid molecules in cytogenetical preparations. Proc Natl Acad Sci USA 1969; 63 : 378-83.

2. Saunders GF, Hsu TC, Getz MJ, et al. Locations of a human satcllitc DNA in human chromosomes. Nature 1972; 236 : 244-6.

3. Jones KW, Prosser J, Corneo G, Ginelli E. The chromosomal location of human satellite DNA III. Chromosoma 1973; $42: 445-51$.

4. Harper ME, Saunders GF. Localization of single copy DNA sequences on G-banded human chromosomes by in situ hybridization. Chromosoma $1981 ; 83: 431-9$.

5. Mattci MG, Philip N, Passage E, Moisan JP, Mandel JL, Mattci JF. DNA probe localization at $18 \mathrm{p} I 13$ by in situ hybridization and identification of a small supernumerary chromosome. Hum Genet 1985; $69: 268-71$.

6. Mattei MG, Baeteman MA, Heilig R, Oberle I, Mandel LJ, Mattci JF. Localization by in situ hybridization of the coagulation factor IX gene and two polymorphic DNA probes with respect to the fragile X site. Hum Genet 1985; $69: 327-31$.

7. Purrello M, Alhadeff B, Esposito D, et al. The human genes for hemophilia $\mathrm{A}$ and hemophilia $\mathrm{B}$ flank the $\mathrm{X}$ chromosome fragile site at $\mathrm{Xq27.3}$. EMBO J 1985; 4 : 725-9.

8. Purrello M, Alhadeff B, Rocchi M, Archidiacono N, Drayna $D$, Siniscalco $M$. Relative position of polymorphic loci of the $\mathrm{X}$ long arm subtelomeric region with respect to the fragile $X$ site. 8 th Human Gene Mapping Workshop, Helsinki, 4-10 août 1985 .

9. Davics KE, Mattci MG, Mattci JF, et al. Linkage studies of $\mathrm{X}$-linked mental retardation: high frequency of recombination in the telomeric region of the human $\mathrm{X}$ chromosome (f ragile site/linkage/recombination/X chromosome). Hum Genet 1985; 70 : 249-55.

I0. Davisson MT, Roderick TH. Linkage map of the mouse. 8th Human Genc Mapping

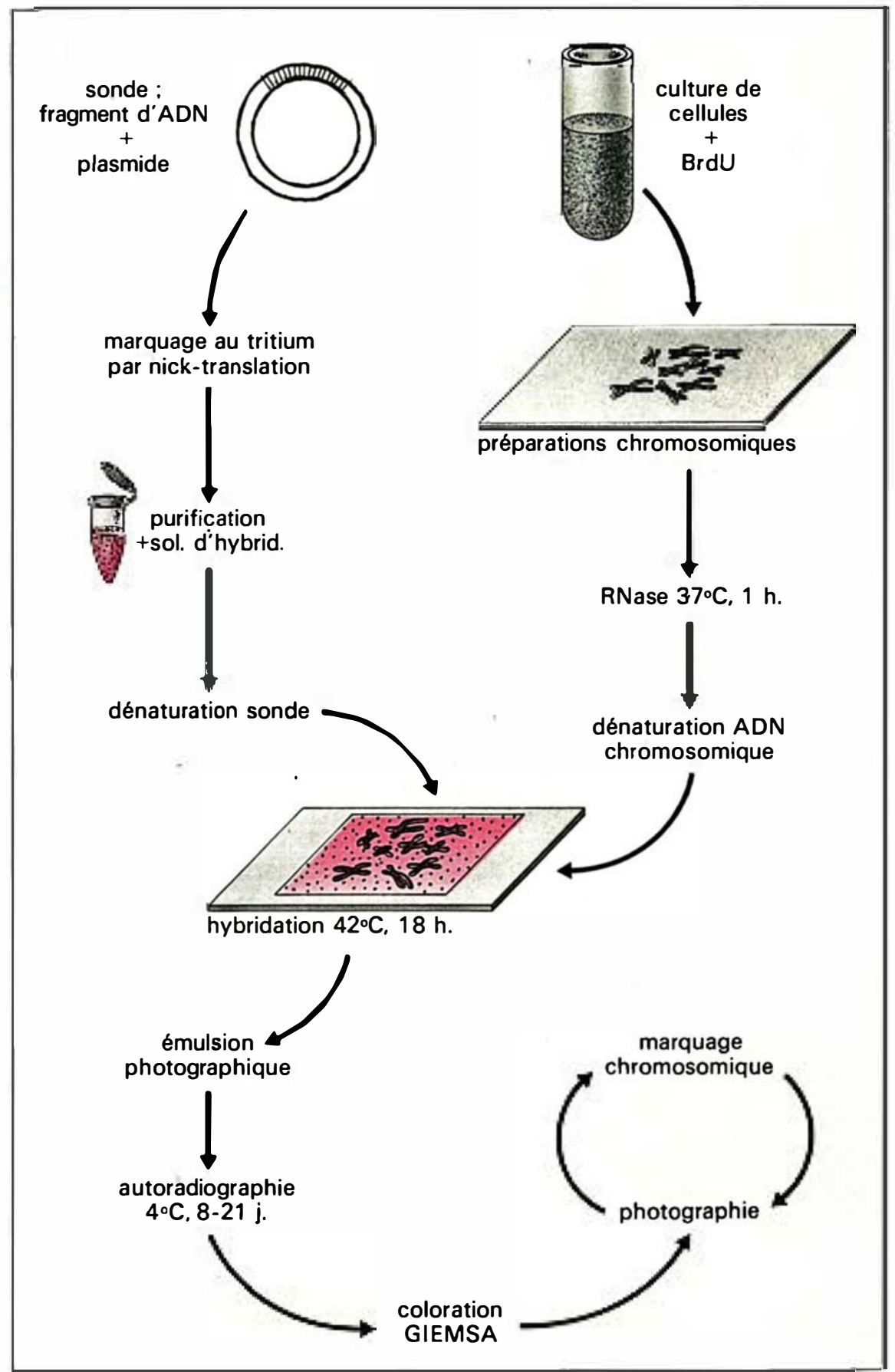

Figure I. Schéma reproduisant les différentes phases de l'hybridation in situ : sol. d'hybrid. : solution d'hybridation; BrdU :5-bromodéoxyuridine; RNase : ribonucléase $A$. 

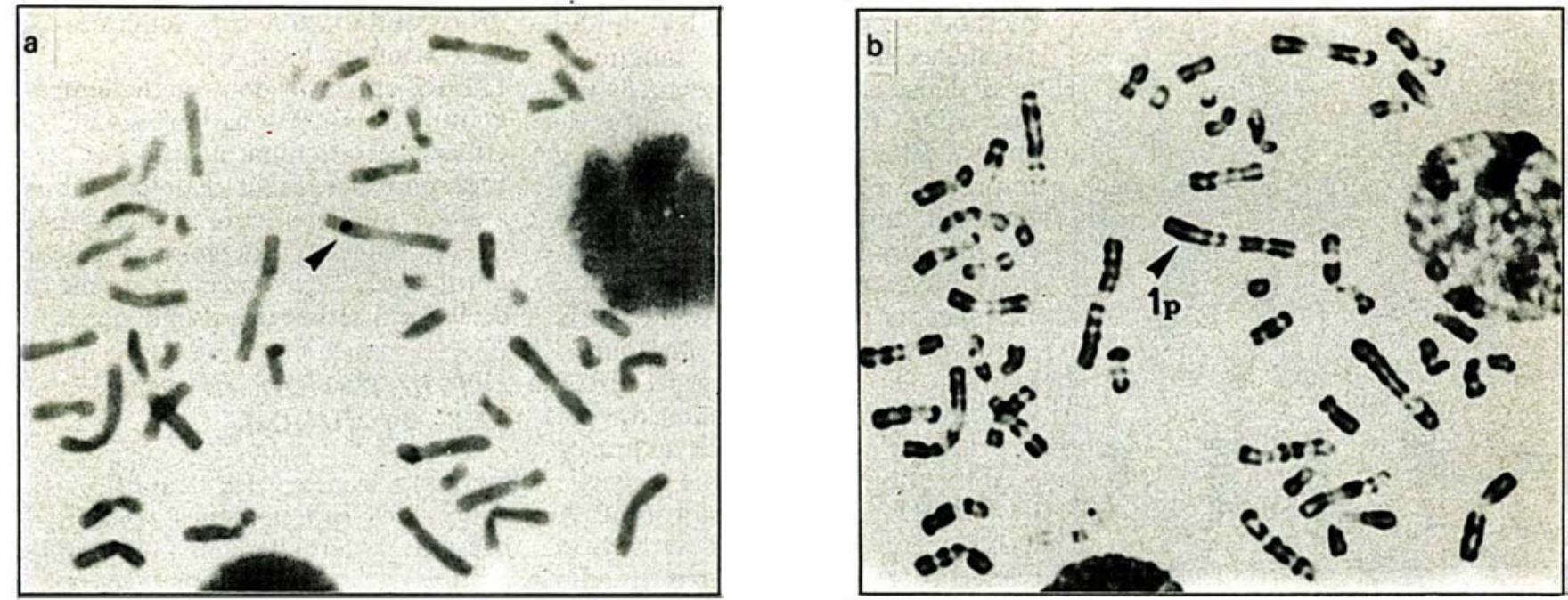

Figure 2. (a) Métaphase colorée au Giemsa après autoradiographie : un grain d'argent est aisément décelable (flèche). (b) La même métaphase après marquage chromosomique : le grain d'argent est localisé précisément sur le bras court d'un chromosome 1.

les sept dernières heures de culture permet d'obtenir ultérieurement un marquage chromosomique de bonne qualité. Les lames portant les métaphases fixées sont traitées par la ribonucléase $\mathrm{A}$ pour éviter toute hybridation non spécifique avec l'ARN endogène. Elles sont ensuite immergées dans la formamide à température adéquate, de telle façon que l'ADN chromosomique, dénaturé, soit accessible à la sonde. Sonde radioactive et préparations chromosomiques ainsi traitées sont mises en contact et incubées à $42^{\circ} \mathrm{C}$ pendant une nuit environ.

\section{Autoradiographie}

Après hybridation, les lames sont recouvertes d'une émulsion photographique constituée d'une gélatine contenant des cristaux de bromure d'argent, susceptibles d'être réduits en argent métallique par le rayonnement $\beta$ du tritium. Les préparations chromosomiques sont alors exposées pendant 8 à 2 I jours à l'abri de la lumière, puis révélées et fixées.

\section{Interprétation des résultats}

Les lames simplement colorées au Giemsa sont observées au microscope et seules sont photographiées les métaphases présentant une bonne dispersion chromosomique et quelques grains d'argent ( $\mathrm{I}$ à 5 au maximum pour une localisation unique). Puis les lames sont traitées de façon à faire apparaître un marquage en bandes sur les chromosomes, pour les identifier sans ambiguïté. Les métaphases précédentes sont photographiées à nouveau et la comparaison des photographies (figure 2) permet d'attribuer chaque grain d'argent à une bande chromosomique précise. La totalité des grains est reportée sur un diagramme représentant un caryotype humain haploïde* ( figure 3).

L'accumulation de points sur une bande chromosomique donnée traduit une forte hybridation entre la sonde et l'ADN chromosomique. Elle correspond donc à une complémentarité maximum et donne la localisation recherchée.

\section{Cartographie génique par HIS}

L'établissement d'une carte génique par l'analyse des hybrides somatiques interspécifiques ou le dosage génique chez des sujets porteurs d'une anomalie chromosomique déséquilibrée présuppose que le gène étudié soit exprimé et son produit bien caractérisé. Par ailleurs, la

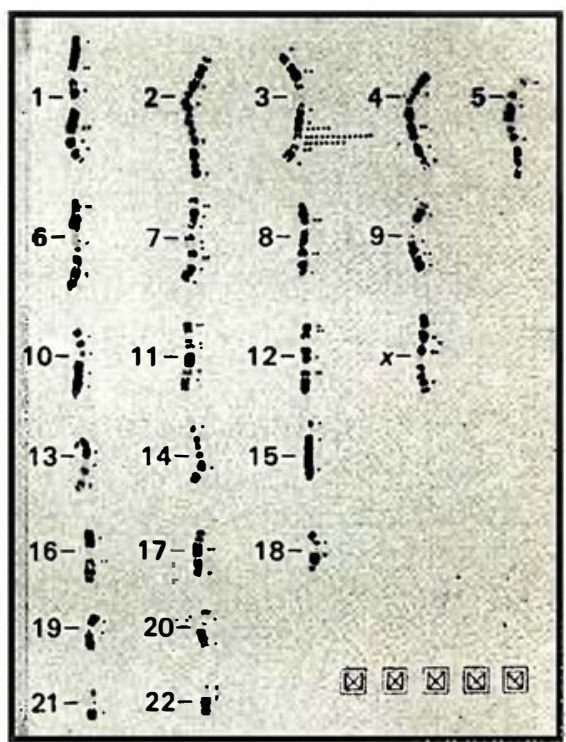

Figure 3. Caryotype humain haploüde sur lequel a été reportée la totalité des grains d'argent trouvés sur 50 métaphases : on peut voir aisément une nette accumulation de grains sur les bras longs du chromosome 3. 


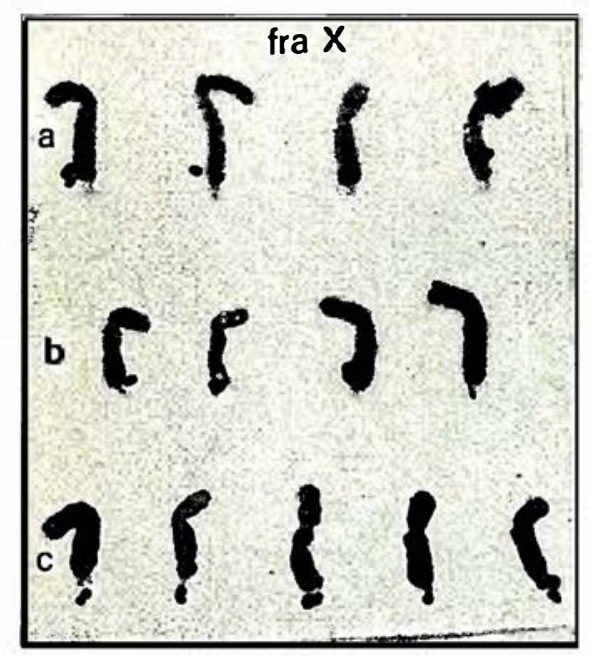

Figure 4. Hybridation in situ de trois sondes localisées sur les bras longs $d u$ chromosome $X$, avec les chromosomes métaphasiques d'un sujet porteur du site fragile fra $\mathrm{Xq27}^{2}$ : (a) La sonde $D X S_{5 I}$ hybride du côté proximal de la cassure. (b) La sonde FIX hybride du côté proximal de la cassure mais plus près du site fragile. (c) La sonde $D X S_{52}$ hybride du côté distal de la cassure.

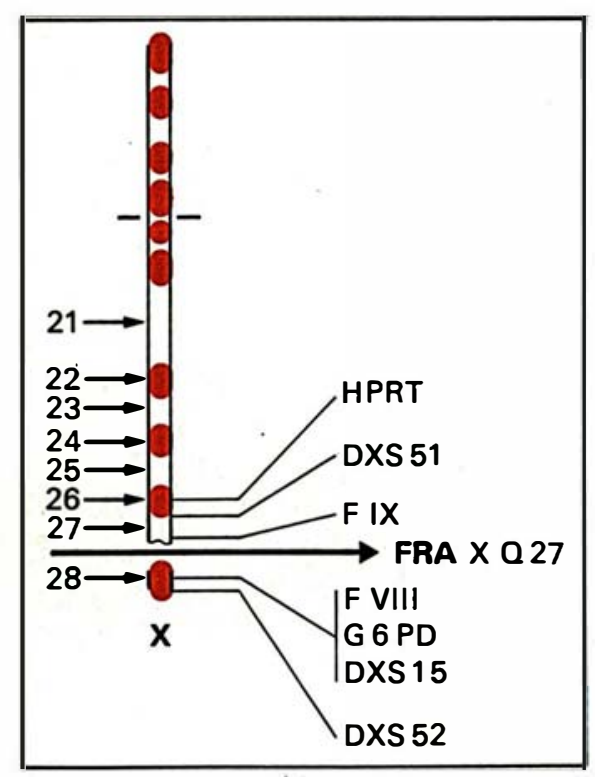

Figure 5. Schéma regroupant les localisations géniques obtenues par différents auteurs, en utilisant l'hybridation in situ sur des chromosomes métaphasi-. ques de sujets porteurs ' $d u$ site fragile fra $X q 27[6,7,8,18,19]$. méthode reposant sur les études familiales de liaison est longue et hasardeuse car elle rcpose sur l'analyse d'un très grand nombre de sujets. Elle reste en outre assez imprécise, le pourcentage de recombinaison entre deux gènes n'étant pas toujours le reflet exact de la distance physique qui les sépare.

Les récents progrès réalisés dans la mise au point de l'HIS en font la méthode la plus séduisante pour localiser de manière rapide, directe et précise n'importe quelle séquence d'ADN. Une cartographie partielle des bras longs du chromosome $\mathrm{X}$ humain, notamment de la région distale contenant le site fragile fra Xq27, a été réalisée par cette méthode. Trois sondes, le gène facteur IX de la coagulation (F IX) et deux sondes polymorphiques, $\operatorname{DXS}_{51}$ (52 A) et DXS 52 (St 14 ), hybridées in situ avec les métaphases d'un sujet normal, ont donné les résultats suivants : localisation dans la bande Xq27 pour les deux sondes F IX et DXS $5 \mathrm{I}$, et dans la bande Xq28 pour la sonde DXS 52 [6]. Puis les trois sondes précédentes ont été hybridées avec les chromosomes mitotiques d'un sujet atteint de retard mental lié à l'X fragile. Ce site fragile s'exprime cytogénétiquement par une cassure entre les bandes Xq27 et Xq28, soit un éloignement physique de ces deux bandes augmentant le pouvoir de résolution de la technique. La figure 4 montre que les deux sondes DXS $5 \mathrm{I}$ et F IX hybrident du côté proximal de la cassure, FIX étant plus proche du site fragile, alors que la sonde DXS 52 hybride du côté distal de la cassure. La cartographie précise des trois sondes sur le chromosome $\mathrm{X}$ est donc finalement :

$$
\begin{gathered}
\text { Xpter } \rightarrow \text { Xcen } \rightarrow \text { Xq26 } \\
\rightarrow \text { DXS }_{51} \rightarrow \text { F IX } \rightarrow \text { fra Xq27 } \\
\text { DXS }{ }_{52} \rightarrow \text { Xqter. }
\end{gathered}
$$

D'autres gènes et sondes polymorphiques ont ainsi été localisés par HIS dans la région subtélomérique* des bras longs du chromosome $\mathrm{X}$ ( figure 5). La comparaison des distances cytogénétiques visualisées par HIS et des distances génétiques basées sur les pourcentages de recombinaison a révélé une distorsion très nette : les loci situés de part et d'autres du site fragile fra $\mathrm{Xq} 27$ ont en effet une fréquence de recombinaison très supérieure à celle attendue $[7,8,9]$.

L'HIS apparaît donc actuellement comme un outil de la biologie moléculaire parfaitement adapté à la cartographie des gènes. Elle contribue aussi à une meilleure connaissance de la structure des gènes, de leur nombre, de l'existence éventuelle et de la localisation des pseudogènes.

\section{Hybridation in situ et évolution}

Certains gènes, peu modifiés au cours des phénomènes évolutifs, présentent actuellement une grande homologie interspécifique, notamment au niveau des séquences codantes ou exons. Il est donc possible d'hybrider in situ une même sonde $A D N_{\mathrm{C}}{ }^{*}$ ne contenant pas d'introns, avec des génomes aussi différents que les génomes humain et murin. La cartographie comparée permet alors de déceler les nombreux remaniements chromosomiques s'étant succédés au cours de l'évolution des espèces. La mise en évidence d'homologies interspécifiques portant non seulement sur des gènes mais sur des familles de gènes ou même des segments chromosomiques, revêt une très grande importance dans la recherche de modèles animaux reproduisant la pathologie humaine. C'est ainsi que la trisomie 2i humaine a trouvé un très bon modèle expérimental dans la trisomie 16 de la souris. On connaît actuellement entre l'homme et la souris une trentaine de régions autosomiques homologues et environ dix gènes situés sur le chromosome X [Io].

\section{Aide au diagnostic cytogénétique}

Les remaniements chromosomiques comme les translocations ou les fragilités constitutionnelles contribuent souvent à préciser les données de l'HIS. Dans certains cas, c'est au contraire l'HIS qui va permettre d'identifier une anomalie chromosomique.

Les petits chromosomes surnuméraires trouvés dans le caryotype de certains sujets retardés mentaux ont généralement une taille trop réduite pour pouvoir être identifiés, malgré 


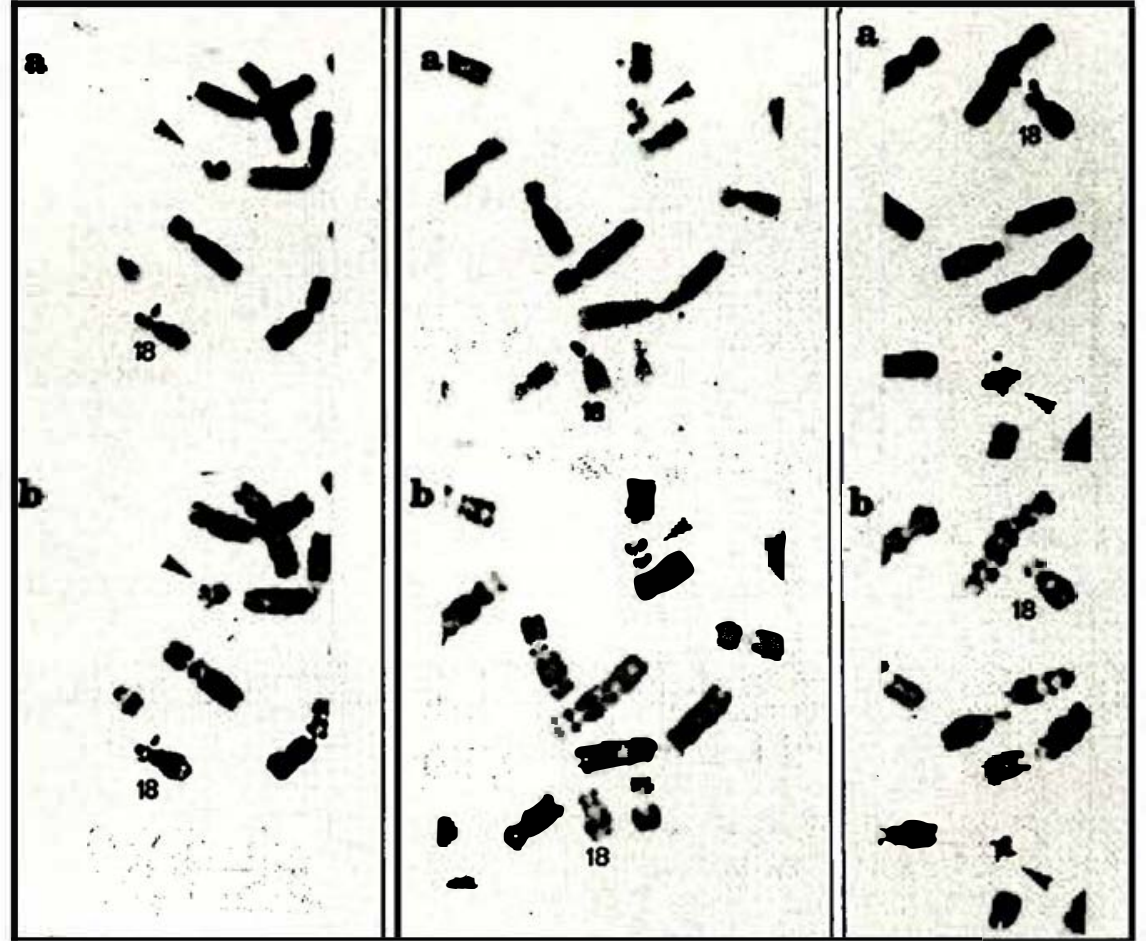

\begin{tabular}{|c|c|c|c|}
\hline PORTEURS D & $\begin{array}{l}\text { YBRIDATION I } \\
\text { VEC LES MÉT } \\
\text { PETIT CHRON }\end{array}$ & $\begin{array}{l}\text { au I } \\
\text { DE.LA SONDE } \\
\text { ES DE TROIS } \\
\text { E MEETACENTF }\end{array}$ & $\begin{array}{l}8 \text { S3 } \\
\text { ETS } \\
\text { IE SURNUMÉRAIRE }\end{array}$ \\
\hline $\begin{array}{c}\text { Nombre de } \\
\text { métaphases } \\
\text { analysées }\end{array}$ & $\begin{array}{c}\text { Nombre total } \\
\text { de grains } \\
\text { d'argent } \\
\text { sur un seul } \\
\text { ou les deux } \\
\text { chromosomes } \\
18 \text { normaux }\end{array}$ & $\begin{array}{c}\text { Nombre total } \\
\text { de grains } \\
\text { d'argent } \\
\text { sur un seul } \\
\text { ou les deux } \\
\text { bras du petit } \\
\text { chromosome } \\
\text { surnuméraire }\end{array}$ & Conclusions \\
\hline $\begin{array}{c}\text { Patient } 1 \\
100\end{array}$ & 53 & 61 & $47, X Y,+i(18 p)$ \\
\hline $\begin{array}{c}\text { Patient } 2 \\
150\end{array}$ & 52 & 58 & $47, X Y,+i(18 p)$ \\
\hline $\begin{array}{c}\text { Patient } 3 \\
150\end{array}$ & 56 & 24 & $\begin{array}{c}47, \mathrm{XY},+\operatorname{del}(18) \\
(\mathrm{q} 123 \rightarrow \text { qter })\end{array}$ \\
\hline
\end{tabular}

* Nombre de métaphases avec un petit chromosome surnuméraire marqué à ses deux extrémités.

Le nombre de grains d'argent trouvés sur le chromosome surnuméraire chez les patients I et 2 permet de l'identifier comme un isochromosome pour les bras courts du I8. Le nombre trouvé sur le chromosome surnuméraire du patient 3 permet de l'identifier comme un chromosome 18 délété pour une partie de ses bras longs.
Figure 6. La sonde $D_{18} S_{3}$ hybride avec les deux extrêmités du petit chromosome surnuméraire chez les patients I et 2 : (a) Trois métaphases partielles colorées au Giemsa après autoradiographie. (b) Les mêmes métaphases après marquage chromosomique.

l'utilisation des techniques de despiralisation chromosomique. L'examen clinique approfondi des patients et l'analyse cytogénétique fine vont, dans un premier temps, orienter le diagnostic vers une trisomie ou une tétrasomie pour une région chromosomique donnée. Dans un deuxième temps, l'HIS d'une sonde caractéristique de ce segment chromosomique conduira à confirmer ou à infirmer l'hypothèse proposée. C'est ainsi que nous avons étudié trois sujets encéphalopathes porteurs d'un petit chromosome métacentrique* surnuméraire chez qui on suspectait une trisomie pour les bras courts du chromosome I8. La sonde Di8 $\mathrm{S}_{3}$ (B 74), préalablement localisée à l'extrémité des bras courts du chromosome I 8 , a été hybridée avec les méthaphases de ces trois patients. Les résultats obtenus sont rapportés dans le tableau I. Chez deux d'entre eux, l'hybridation de Di8 $8 S_{3}$ aux deux extrémités du petit chromosome surnuméraire permet de penser que celui-ci est un isochromosome* pour les bras courts d'un chromosome 18 , c'est-à-dire une tétrasomie $\mathrm{I} 8 \mathrm{p}$ (figure 6). 


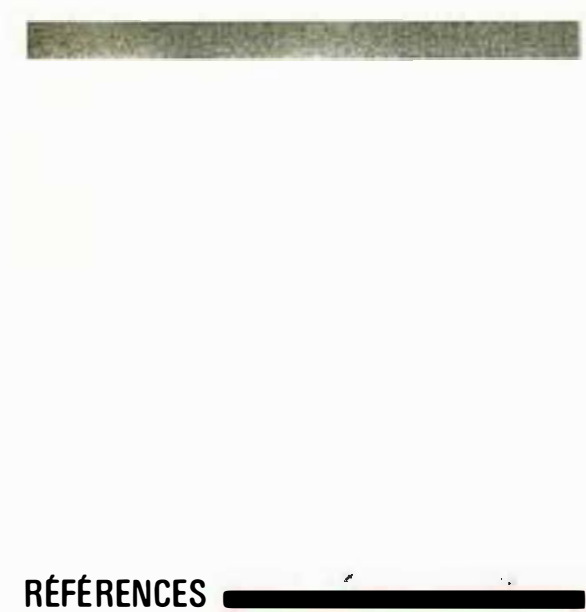

11. Magenis RE, Dermid H, Sheehy R. The extra chromosome in Cat-Eye syndrome is derived from chromosome 22; evidence from in situ hybridization of a chromosome 22 specific DNA probe. 8th Human Gene Mapping Workshop, Helsinki, 4-1o août 1985

12. Mattei MG, Passage E, Julien C, et al. Chromosome 22 is involved in Cat-Eye syndrome as demonstrated by in situ hybridization. 8th Human Gene Mapping Workshop, Helsinki, 4-10 août 1985 .

13. Buckle V, Boyd Y, Craig MW, Frazer N, Goodfcllow PN, Wolfe J. Localization of Y chromosomal sequences in normal and "XX " males. 8th Human Gene Mapping Workshop, Helsinki, 4-10 août 1985

14. Magenis RE, Sheehy R, Olson S, Brown MG, Casanova M, Fellous $M$. Genes for maleness localized to distal onc-half of $Y$ chromosome short arm : cvidence from in situ hybridization of a Y-derived single copy DNA probe. 8th Human Gene Mapping Workshop, Helsinki, 4-10 août I985.

15. Mattei MG, Mattei JF, Vidal I, Giraud F. Structural anomalies of the $\mathrm{X}$ chromosome and inactivation center. Hum Genet 1981; $56: 401-8$.

16. Dalla-Favera R, Bregni M, Erikson J, Patterson D, Gallo RC, Crocc CM. Human c-myc oncogene is located on the region of chromosome 8 that is translocated in Burkitt lymphoma cells. Proc Natl Acad Sci USA 1982; 79 : 7824-7.

17. Schwab M, Ellison J, Busch M, Rosenau W, Varmus HE, Bishop JM. Enhanced expression of the human gene $\mathrm{N}$-myc consequent to amplification of DNA may contribute to malignant progression of neuroblastoma. Proc Natl Acad Sci USA 1984; 81 : 4940-4.

18. Buckle V, Craig JW, Edwards JH. Fine assignment of the coagulation F IX gene. 8th Human Gene Mapping Workshop, Helsinki, 4-10 août I985.

19. Szabo P, Purrello M, Rocchi M, et al. Cytological mapping of the human glucosc-6phosphate deshydrogenase gene distal to the fragiie X site siggests a high rate of meiotic recombination across this site. Proc Natl Acad Sci USA $1984 ; 81: 7855-9$.

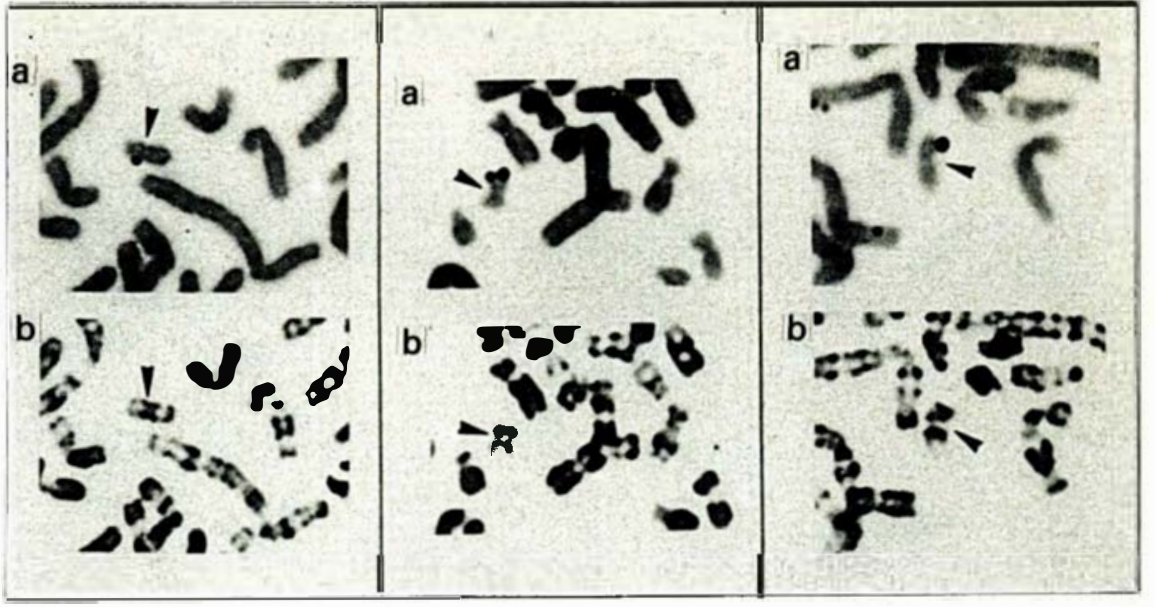

Figure 7. La sonde $D_{I} 8 S_{3}$ hybride avec une seule extrêmité du petit chromosome surnuméraire chez le patient $3:$ (a) Trois métaphases partielles colorées au Giemsa après autoradiographie. (b) Les mêmes métaphases après marquage chromosomique.

Chez le troisième sujet, la sonde $\mathrm{D}_{1} 8 \mathrm{~S}_{3}$ donne un signal positif sur une seule extrémité du petit chromosome métacentrique surnuméraire, indiquant une trisomie pour un chromosome i 8q- métacentrique (figure 7 ).

D'autre part, l'HIS de sondes adaptées a confirmé que le petit chromosome surnuméraire associé au syndrome de l'Oeil-de-Chat dérivait du chromosome 22 , le surdosage chromosomique pouvant être soit une trisomie, soit une tétrasomie pour la région proximale des bras longs du chromosome 22 [I I, I2].

Une autre application de l'HIS est le diagnostic des anomalies inframicroscopiques, c'est-à-dire de séquences d'ADN inférieures à 3000 kilobases (taille approximative d'une superbande). Il a été ainsi démontré que certains mâles " $\mathrm{XX}$ " et certains hermaphrodites à caryotype 46,XX étaient porteurs de séquences $\mathrm{Y}$-spécifiques à l'extrémité des bras courts d'un chromosome $\mathrm{X}$, au niveau de la zone d'appariement méiotique entre les chromosomes $X$ et $Y[13,14]$.

L'identification des anomalies du chromosome $\mathrm{X}$ reste souvent ambiguë, non seulement à cause de l'inactivation de l'un des chromosomes $\mathrm{X}$ qui rend les dosages enzymatiques inadaptés, mais aussi parce que l'existence de mosaïques rend hasardeuses les hybridations sur blot. L'HIS de sondes spécifiques est actuellement la meilleure méthode pour démontrer l'existence, ou la non existence, des isochromosomes pour les bras courts du chromosome $\mathrm{X}$; les quelques cas d'isochromosomes $\mathrm{Xp}$ publiés ne sont en effet convaincants, ni sur le plan clinique, ni d'un point de vue cytogénétique [ 15 ].

\section{Étude des cancers}

Toutes les cellules humaines contiennent des gènes susceptibles de produire des cancers : les oncogènes. Ces gènes, qui interviennent normalement dans le contrôle de la prolifération et de la croissance, voire de la différenciation cellulaire, peuvent soudainement être activés et devenir source de transformation cancéreuse. L'activation d'un oncogène cellulaire peut être liée à une altération de sa structure (mutation ponctuelle) ou encore à un changement dans sa localisation. Dans ce cas, une séquence stimulatrice peut, à la suite d'un réarrangement chromosomique, se trouver placée à proximité d'un oncogène et donc renforcer son expression. L'HIS a permis de mettre en évidence une remarquable concordance entre la 
localisation chromosomique des oncogènes cellulaires et les points de cassure impliqués dans les remaniements chromosomiques spécifiques de certains cancers (ex. : oncogène c- myc et translocation entre un chromosome 8 et un chromosome I4 dans le lymphome de Burkitt [16]).

Dans un certain nombre de cancers, l'activation d'un oncogène cellulaire est liée à un mécanisme d'amplification (ex. : amplification de l'oncogène $\mathrm{N}$-myc dans le neuroblastome [17]). La cellule contient alors un grand nombre de copies actives apparaissant sous forme de chromosomes acentriques "double minute " (DM), ou de régions colorées uniformément (HSR) et responsables de l'expression augmentée de l'oncogène.

L'HIS s'avère alors indispensable pour étudier les caractéristiques de cette amplification, qu'elles soient qualitatives ou quantitatives.

\section{Conclusion}

L'HIS permet de localiser n'importe quelle séquence de nucléotides sur des chromosomes fixés en métaphase. Elle s'avère actuellement indispensable pour l'établisse- ment de la carte génique humaine, l'étude des différents mécanismes de l'évolution, le diagnostic chromosomique et l'analyse des processus cancéreux. Cette technique récente constitue un point de convergence entre le domaine de la cytogénétique, dont la définition minimale est d'environ 3000 kilobases et celui de la biologie moléculaire, dont le niveau d'appréhension maximum est de 50 kilobases.

L'utilisation de sondes non radioactives (sondes froides), marquées par des fluorochromes ou des enzymes colorimétriquement décelables, devrait bientôt permettre à l'HIS d'élargir son domaine d'applications. Ces sondes présentent en effet beaucoup d'avantages : elles sont chimiquement stables et donnent des résultats très reproductibles. La détection des sites d'hybridation est plus rapide que par autoradiographie ( $I$ jour contre I 5 environ avec les sondes radio-actives). Ces sondes sont par ailleurs d'un emploi plus facile. Leur pouvoir de résolution n'atteint pas cependant celui des sondes radio-actives, et l'hybridation in situ de sondes froides ne permet pas encore la localisation de séquences uniques de petite taille (taille inférieure à 20 kilobases)

\section{* GLOSSAIRE*}

ADN satellite : $A D N$ haurement répétitif, c'est-à-dire constitué d'une séquence de bases répétée en tandem un très grand nombre de fois dans le génome.

Chromosome polytène : chromosome généré par des replications successives, sans séparation ultérieure des copies qui restent à l'intérieur d'une seule structure.

Hybridation : réassociation de chaînes d'acides nucléiques complémentaires.

Nick translation : procédé permettant le marquage interne d'un $A D N$ double brin. Il s'agit de la coupure d'un brin par la DNase, suivie de sa réparation par l'ADN polymérase avec des nucléotides radio-actifs.
Caryotype haploïde : caryotype comprenant un seul exemplaire de chaque chromosome.

Région subtélomérique : région presque terminale des bras d'un chromosome.

$A D N_{c}$ ou $A D N$ complémentaire : copie double brin synthétisée in vitro à partir de l'ARN et ne contenant donc que des exons.

Chromosome métacentrique : chromosome dont le bras court " $p$ " a la même taille que le bras long " $q$ ».

Isochromosome : chromosome résultant d'une mauvaise division du centromère et constitué de deux bras identiques : deux fois le bras court, ou deux fois le bras long.

\section{Summary}

In situ hybridization, previously limited to detection of repetitive gene families, now permits direct localization of any given single-copy DNA sequence.

This technique is now feasible for refined gene mapping and has become very helpful in cytogenetic diagnosis and detection of infra-microscopic chromosomal abnormalities. It is also essential for studying the various process of oncogene activation in the development of cancer and analysing complex mechanisms implicated in species evolution.

\section{TIRES A PART}

M G. Mattei : Inserm U 242 ct Centre de génétique médicale, Hôpital d'enfants de la Timonc, 13385 Marseille cedex 5 . 